\title{
A distal single nucleotide polymorphism alters long-range regulation of the PU.1 gene in acute myeloid leukemia
}

\author{
Ulrich Steidl,, ${ }^{1}$ Christian Steidl, 2,3 Alexander Ebralidze, ${ }^{1}$ Björn Chapuy, ${ }^{2}$ Hye-Jung Han, ${ }^{4}$ \\ Britta Will,, ${ }^{1,5}$ Frank Rosenbauer, ${ }^{1,6}$ Annegret Becker, ${ }^{2}$ Katharina Wagner, ${ }^{1,7}$ Steffen Koschmieder, ${ }^{1,8}$ \\ Susumu Kobayashi, ${ }^{1}$ Daniel B. Costa, ${ }^{1}$ Thomas Schulz, ${ }^{2}$ Karen B. O'Brien, ${ }^{1}$ Roel G.W. Verhaak, ${ }^{9}$ \\ Ruud Delwel, ${ }^{9}$ Detlef Haase, ${ }^{2}$ Lorenz Trümper, ${ }^{2}$ Jürgen Krauter, ${ }^{7}$ Terumi Kohwi-Shigematsu, ${ }^{4}$ \\ Frank Griesinger, ${ }^{2}$ and Daniel G. Tenen 1
}

\begin{abstract}
${ }^{1}$ Harvard Institutes of Medicine, Harvard Medical School, and Harvard Stem Cell Institute, Boston, Massachusetts, USA. 2Department of Hematology and Oncology, Georg-August University of Göttingen, Goettingen, Germany. ${ }^{3}$ Department of Pathology, British Columbia Cancer Agency, Vancouver, British Columbia, Canada. ${ }^{4}$ Life Sciences Division, Lawrence Berkeley National Laboratory, Berkeley, California, USA. ${ }^{5}$ Department of Cell Biology, University of Freiburg, Freiburg, Germany. ${ }^{6}$ Max Delbrück Center for Molecular Medicine, Berlin, Germany. ${ }^{7}$ Department of Hematology, Hemostasis and Oncology, Hannover Medical School, Hannover, Germany. ${ }^{8}$ Department of Medicine, Hematology and Oncology, University Hospital Münster, Muenster, Germany. ${ }^{9}$ Department of Hematology,
\end{abstract} Erasmus University Medical Center, Rotterdam, The Netherlands.

\begin{abstract}
Targeted disruption of a highly conserved distal enhancer reduces expression of the PU.1 transcription factor by $80 \%$ and leads to acute myeloid leukemia (AML) with frequent cytogenetic aberrations in mice. Here we identify a SNP within this element in humans that is more frequent in AML with a complex karyotype, leads to decreased enhancer activity, and reduces PU.1 expression in myeloid progenitors in a developmentdependent manner. This SNP inhibits binding of the chromatin-remodeling transcriptional regulator special AT-rich sequence binding protein 1 (SATB1). Overexpression of SATB1 increased PU.1 expression, and siRNA inhibition of SATB1 downregulated PU.1 expression. Targeted disruption of the distal enhancer led to a loss of regulation of PU.1 by SATB1. Interestingly, disruption of SATB1 in mice led to a selective decrease of PU.1 RNA in specific progenitor types (granulocyte-macrophage and megakaryocyte-erythrocyte progenitors) and a similar effect was observed in AML samples harboring this SNP. Thus we have identified a SNP within a distal enhancer that is associated with a subtype of leukemia and exerts a deleterious effect through remote transcriptional dysregulation in specific progenitor subtypes.
\end{abstract}

\section{Introduction}

A tightly regulated network of transcription factors is critical for normal hematopoiesis. The lineage-specific transcription factor PU.1 is essential for myeloid development, and its disruption leads to block of myeloid and B cell development as well as defective function of HSCs, including a block in differentiation to common myeloid progenitors and common lymphoid progenitors (1-4). PU.1 serves multiple roles during normal hematopoiesis, including acting as a transcriptional regulator of target genes and as an inhibitor of other transcriptional regulators, often through protein-protein interactions (reviewed in refs. 5, 6). Even moderate decreases of PU.1 levels can lead to disturbed gene expression, abnormal cytokine signaling, and hyperproliferation of progenitor populations, genomic instability, and methylation of tumor suppressor genes, all of which likely contribute to malignant transformation of myeloid and lymphoid precursors (refs. 7-9, reviewed in ref. 10). In addition, multiple other studies have confirmed that dysregulation of PU.1 function contributes to development of AML in the mouse, demonstrating its role as a tumor

Nonstandard abbreviations used: AML, acute myeloid leukemia; CMP, common myeloid progenitor; GMP, granulocyte-macrophage progenitor; MEP, megakaryocyteerythrocyte progenitor; URE, upstream regulatory element.

Conflict of interest: The authors have declared that no conflict of interest exists. Citation for this article: J. Clin. Invest. 117:2611-2620 (2007). doi:10.1172/JCI30525 suppressor (11-14). Combined, these studies demonstrate that precise regulation of PU.1 expression levels is essential to maintain normal hematopoiesis and prevent the development of leukemia. We have recently reported that the transcriptional control of PU.1 gene expression is mediated by a distal upstream regulatory element (URE) that is highly conserved among multiple species including mice and humans $(15,16)$. This URE appears to have a specific effect on PU.1 gene regulation; targeted disruption of this element affects the expression of solely PU.1 but not other genes in the upstream or downstream genomic neighborhood (9). Mice lacking the URE have $80 \%$ reduced expression of PU.1 in the $\mathrm{BM}$ and develop acute myeloid leukemia (AML) (7). Also, reduced PU.1 expression levels have been observed in HSCs and granulocyte-macrophage progenitors (GMPs) in patients with AML (ref. 9 and U. Steidl and D.G. Tenen, unpublished data). Therefore we hypothesized that mutations within the URE could cause dysregulation of PU.1 and thus serve as a potential mechanism contributing to leukemogenesis in human AML. In this study we analyzed the URE in patients with AML by means of FISH and direct sequencing. We identified a SNP within the URE, which occurs more frequently in AML with complex karyotype. We identify the chromatin-remodeling special AT-rich sequence binding protein 1 (SATB1) as a novel long-range regulator of PU.1 and show that the SNP in the distal URE critically alters the PU.1-regulatory function of SATB1 in a development-dependent manner. 
A

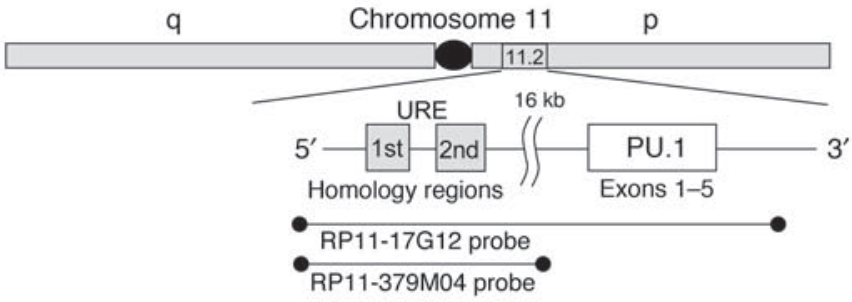

B

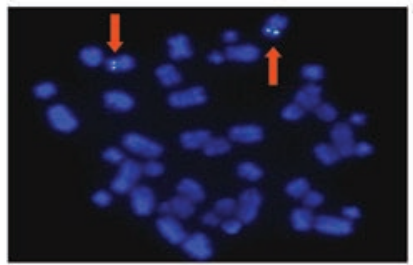

C
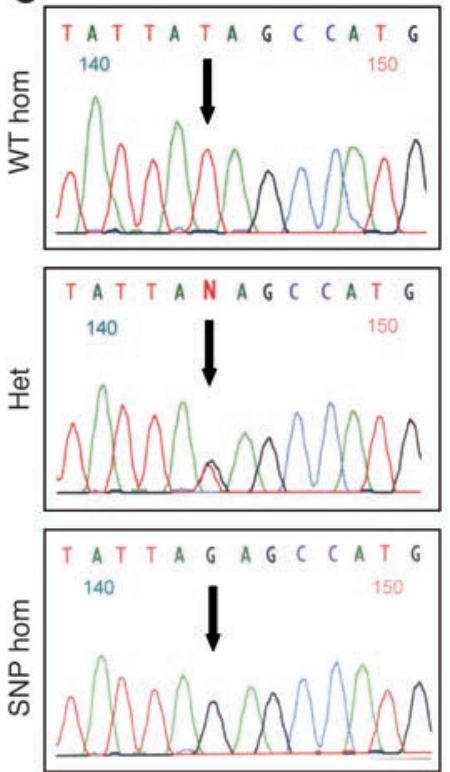

$\mathbf{E}_{60}$ SNP in 1st homology region

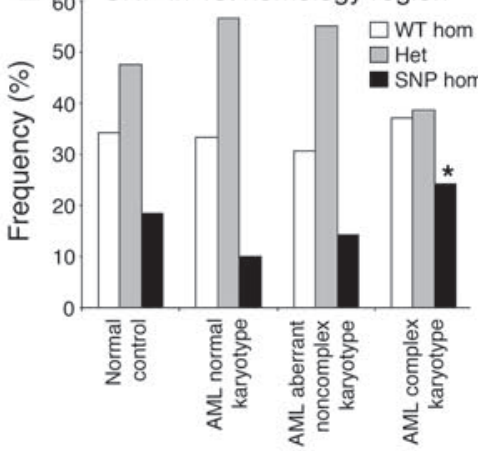

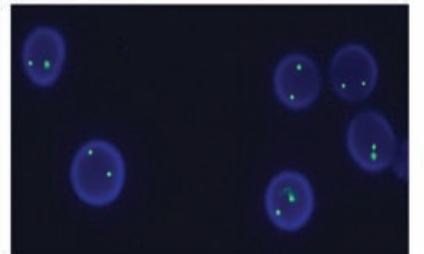

D
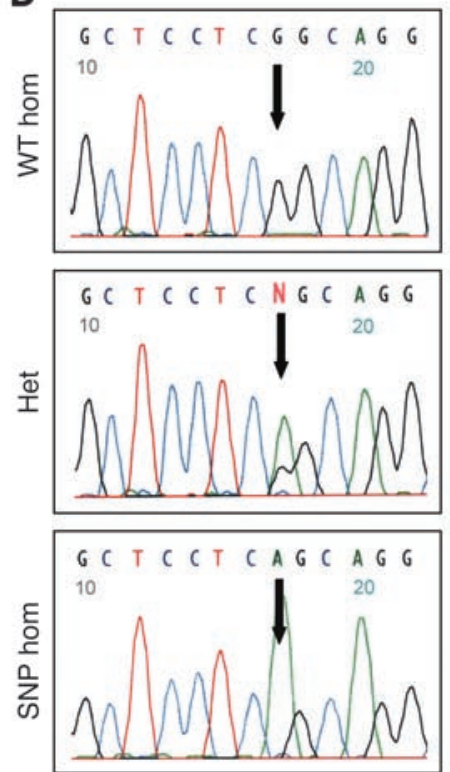

F ${ }_{90}$ SNP in 2nd homology region

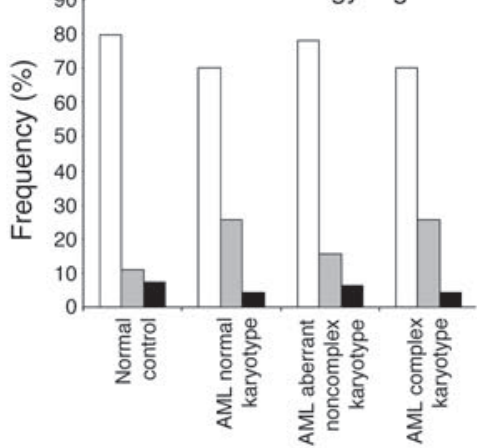

\section{Figure 1}

Genomic analysis of distal URE of PU.1. (A) Schematics of genomic locus of human PU.1 gene including its 5 exons (white box) and -16-kb URE consisting of 2 highly conserved homology regions (gray boxes). Localization of the probes for fluorescence in situ hybridization (RP11-17G12 and RP11-379M04) is indicated by lines with filled circles at the ends. The long $(q)$ and short ( $p$ ) arms of chromosome 11 and the band 11.2 are indicated. (B) Fluorescence in situ hybridization with probe RP11-379M04 covering the URE locus. Left: Metaphase FISH showing 2 signals on each chromosome 11 (arrows). Right: Interphase FISH of 1 representative of 80 patients with 2 signals per cell. (C) Direct sequencing identifies SNP in the first homology region of PU.1 URE. Representative sequencing traces of patients with wild-type site (WT hom), heterozygous site (het), and homozygous SNP (SNP hom) shown. (D) Identification of a SNP in the second homology region of URE. Representative sequencing graphs of patients with wild-type site, heterozygous site, and homozygous SNP shown. (E) Higher abundance of the homozygous SNP in first homology region in patients with AML with complex karyotypes. Bar diagram shows SNP status of normal controls and AML with normal karyotype, with aberrant noncomplex karyotype, and with complex karyotype. ${ }^{*} P=0.027\left(\chi^{2}\right)$ and $P=0.018$ (Fisher's exact); odds ratio, 2.9; odds ratio $95 \%$ confidence interval, 1.22-6.83. (F) Frequency of SNP in the second homology region of URE is not different between normal controls or AML.

chromosome 11 (Figure 1A). It consists of 2 highly conserved regions (first and second homology regions) 16 $\mathrm{kb}$ upstream of the PU.1 transcriptional start site. We performed FISH analysis of 80 patients with AML to check for deletions in this region. We utilized 2 probes, one corresponding to the URE region and another that also included the PU.1 gene locus (Figure 1A). Neither probe showed signal losses in any of the examined 80 patients (Figure 1B), demonstrating that deletion of this region does not frequently occur in patients with AML. To determine whether point mutations in the URE can be observed in AML patients, we examined the 2 highly conserved regions within the URE by direct sequencing. We sequenced genomic DNA of 209 patients with AML and 158 healthy controls and identified 2 base changes, one in the first homology region and the other in the second homology region (Figure 1, C and D). Nonhematopoietic tissue in individual patients showed the same base changes and was always similarly homozygous $(n=6)$, heterozygous $(n=3)$, or wild type $(n=3)$ in corresponding hematopoietic and nonhematopoietic specimens within the same patient sample. This demonstrates that the detected base changes represent germline SNPs. The overall fre-

\section{Results}

Identification of a SNP in the distal enhancer of PU.1 that is more frequent in AML with complex karyotype. Recently, we have demonstrated that knockout of a distal enhancer of PU.1 leads to a knockdown of PU. 1 expression to $20 \%$ of normal levels and induces AML with frequent cytogenetic aberrations in mice (7). This finding prompted us to examine the genomic locus of this URE of PU.1 in patients with AML. In humans, the URE is located on the short arm of quency of the homozygous SNPs in the first homology region was not significantly changed in AML patients compared with healthy controls (Table 1). However, this SNP was 2.4-fold more frequent in patients with AML with complex karyotype than in AML with normal karyotype ( $\chi^{2}$ test, $P=0.027$; Fisher's exact test, $P=0.018$ ) (Table 1 and Figure 1E). We did not find a change of frequency of the SNP in the second homology region in complex karyotypic AML (Table 1 and Figure 1F). The finding that the SNP within the first 


\section{Table 1}

Frequency of the SNP in the first and second homology regions of the URE of PU.1 in patients with $A M L$ and in healthy control subjects

\begin{tabular}{lcccc}
\hline Parameter & $\begin{array}{c}\text { Homozygous } \\
\text { WT }\end{array}$ & $\begin{array}{c}\text { Heterozygous } \\
\text { SNP/WT }\end{array}$ & $\begin{array}{c}\text { Homozygous } \\
\text { SNP }\end{array}$ & Total \\
SNP, first homology region, no. (\%) & & & & \\
Healthy control & $54(34.2)$ & $75(47.5)$ & $29(18.4)$ & $158(100)$ \\
AML, total & $71(34.0)$ & $105(50.2)$ & $33(15.8)$ & $209(100)$ \\
AML, normal karyotype & $30(33.3)$ & $51(56.7)$ & $9(10.0)$ & $90(100)$ \\
AML, aberrant noncomplex karyotype & $15(30.6)$ & $27(55.2)$ & $7(14.3)$ & $49(100)$ \\
AML, complex karyotype & $26(37.1)$ & $27(38.6)$ & $17(24.3)^{A}$ & $70(100)$ \\
SNP, second homology region, no. (\%) & & & & \\
Healthy control & $43(81.1)$ & $6(11.3)$ & $4(7.5)$ & $53(100)$ \\
AML, total & $85(77.3)$ & $20(18.2)$ & $5(4.5)$ & $110(100)$ \\
AML, normal karyotype & $33(70.2)$ & $12(25.5)$ & $2(4.3)$ & $47(100)$ \\
AML, aberrant noncomplex karyotype & $25(78.1)$ & $5(15.6)$ & $2(6.3)$ & $32(100)$ \\
AML, complex karyotype & $27(87.1)$ & $3(9.7)$ & $1(3.2)$ & $31(100)$ \\
\hline
\end{tabular}

Different cytogenetic subgroups are indicated. Absolute numbers are shown. ${ }^{A} P=0.027\left(\chi^{2}\right)$ and $P=0.018$ (Fisher's exact); odds ratio, 2.9; odds ratio 95\% confidence interval, 1.22-6.83.

ate long-range transcriptional regulation. Given that the URE as well as its upstream and downstream regions harbors several enriched As, Ts, and Cs in sequence, we hypothesized that the DNA binding protein SATB1 might mediate long-range transcriptional regulation of PU. 1 by binding to the URE. SATB1 plays an important role in chromatin remodeling and serves as a transcriptional regulator by folding chromatin into loop domains and tethering DNA elements to a cage-like SATB1 network, which enhances the formation of protein-DNA complexes between distal elements $(17,18)$. SATB1 binds to special AT-rich sequences in which one strand consists of mixed As, Ts, and Cs, excluding Gs. SATB1 binding is greatly reduced when this feature is destroyed by mutation (19). To test this hypothesis, we performed chromatin immunoprecipitation of chromatin isolated from myeloid leu-

homology region correlates with AML with complex karyotype suggests that this SNP may play a role in disease progression of AML. As knockout of the URE results in the development of AML with frequent genetic aberrations (7), we examined whether this SNP is functionally relevant for the enhancer function of the URE.

The SNP reduces the enhancer activity of the URE of PU.1. We have recently shown that the PU.1 URE in combination with the PU.1 promoter induces expression of a reporter gene in vivo, while the promoter alone is not sufficient $(15,16)$. To determine whether the SNP in the URE changes the enhancer activity of the URE, we utilized luciferase reporter constructs of the promoter alone, wildtype URE plus promoter, and point-mutated URE plus promoter (Figure 2A). After stable transfection into U937 cells, we found that the mutated URE had significantly less enhancer activity compared with wild-type URE (Figure 2, B and C) in single clones (4.5-fold; $P<0.001$ ) as well as in pools (3.9-fold; $P<0.001)$, demonstrating the functional relevance of the point mutation.

Binding of SATB1 to the URE is disrupted by the SNP. We next sought to identify potential binding factors at this site that may medi-

\section{Figure 2}

The SNP in the first homology region of the URE of PU.1 leads to reduced enhancer activity. (A) Schematics of the reporter constructs utilized for stable transfections of U937 myeloid cells. Top: The proximal promoter of PU.1 in the pXP2 luciferase vector. Middle: The wildtype URE plus the proximal promoter of PU.1. Bottom: The SNP URE plus the proximal promoter of PU.1. The point mutation representing the SNP is indicated by a star. (B and $\mathbf{C})$ Luciferase reporter assays after stable transfection of the above-described constructs into U937 cells shows reduced enhancer activity of the point-mutated URE. (B) The mean luciferase activity of 3 independent clones is displayed. Error bars indicate SD. (C) The mean luciferase activity of 3 independent cell pools is shown. Luciferase activity was normalized to transgene copy number as determined by Southern blotting. Error bars indicate SD. ${ }^{*} P<0.001$. kemic U937 and HL60 cells. Both U937 and HL60 carry homozygous wild-type alleles at the site of the SNP (data not shown). We found that SATB1 binds to the URE in both cell types in vivo (Figure 3A). To address whether the base change of the SNP alters SATB1 binding, we performed EMSAs with nuclear extracts of U937 cells. Binding of the SATB1 complex to the labeled wild-type probe was subject to competition from the wild-type oligonucleotide itself or an oligonucleotide carrying a known SATB1 binding site in the IgH promoter (17). In contrast, a probe containing the SNP led to a $70 \%(P=0.002 ; n=3)$ reduction of binding of the SATB1 complex compared with the wild-type probe (Figure 3B).

$S A T B 1$ is a positive regulator of PU.1 expression in myeloid cells. To assess whether SATB1 regulates PU.1 expression in myeloid cells, we studied the effects of both the inhibition and overexpression of SATB1 in myeloid leukemic U937 cells. We stably transfected an expression construct that expresses a SATB1-directed siRNA along with a neomycin resistance gene into U937 cells. A construct carrying the neomycin resistance cassette alone served as a control. The SATB1 siRNA construct decreased SATB1 mRNA expression 3.6-fold 


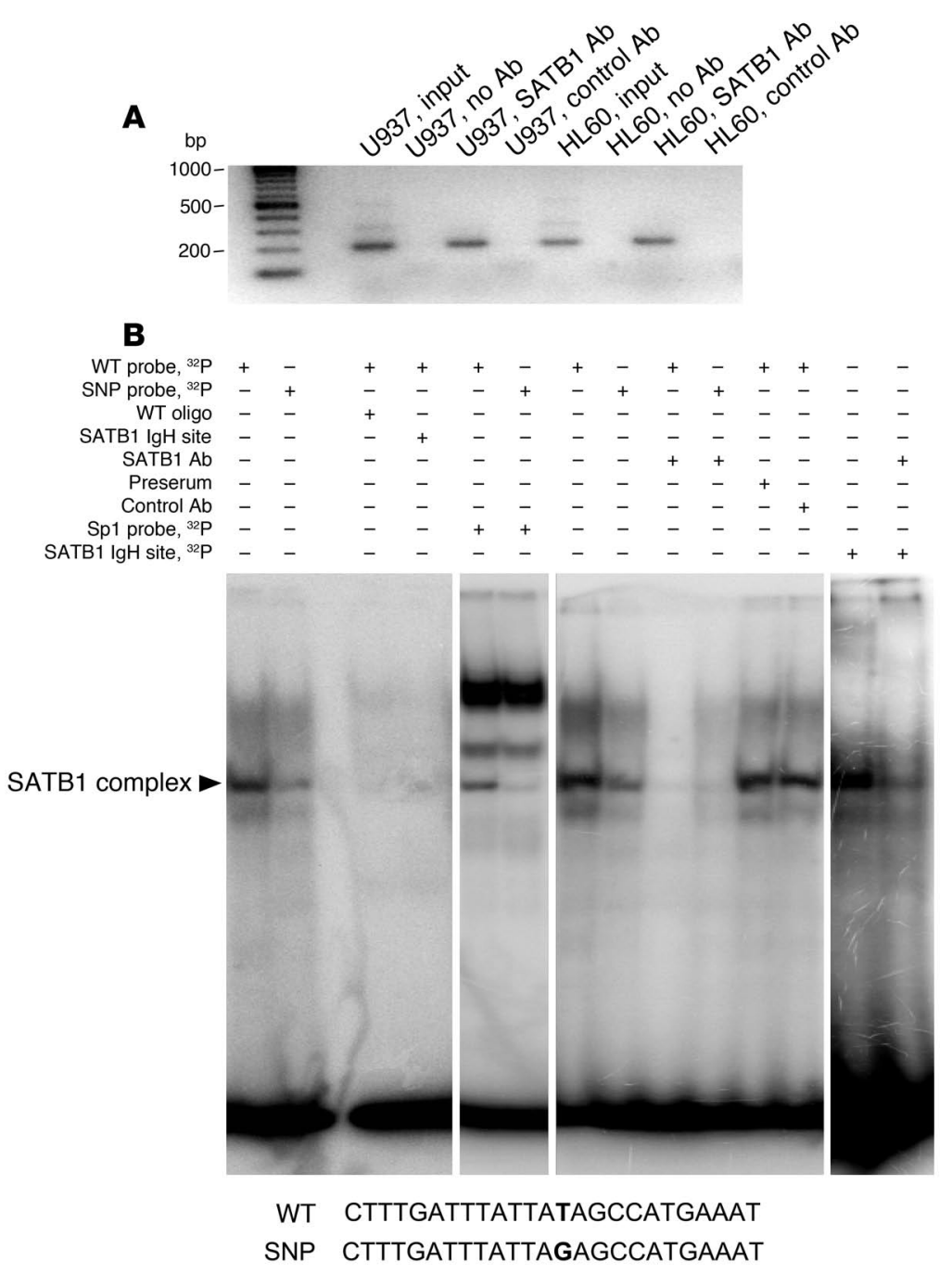

\section{Figure 3}

The SNP in the first homology region of the URE of PU.1 diminishes binding of SATB1. (A) Chromatin immunoprecipitation shows SATB1 binding to the URE in U937 and HL60 cells. The genomic region of the putative SATB1 binding site was PCR amplified after reverse crosslink of the immunoprecipitates. An input control and precipitates utilizing a SATB1 antibody, no antibody, or a nonspecific control antibody are shown. PCR products were verified by sequencing. (B) EMSA utilizing nuclear extracts of U937 cells and gel-purified probes (WT probe, ${ }^{32} \mathrm{P}$, and SNP probe, ${ }^{32} \mathrm{P}$ ) covering the SATB1 binding site are shown. The wild-type probe (WT oligo) and a previously described SATB1 binding probe (SATB1 lgH site) were used for competition. A SATB1 antibody was used for supershift. A labeled Sp1 binding probe served as a loading control in 2 lanes. The ${ }^{32} \mathrm{P}$-labeled SATB1 IgH site served as positive control. The presence $(+)$ or absence $(-)$ of the respective reagents is indicated for each lane. The probes are shown below the gel.
$(P=0.02)$ and led to a 2.6-fold reduction of PU.1 mRNA expression $(P=0.03)$ (Figure 4A). Expression of transcription factor CCAAT/ enhancer binding protein- $\gamma(\mathrm{C} / \mathrm{EBP} \gamma)$ remained unchanged, suggesting the specificity of the SATB1-directed siRNA (Figure 4A). However, off-target effects cannot be completely ruled out. Western blotting showed an even greater effect at the protein level. siRNA reduced SATB1 by 6.6-fold and led to a 4.3-fold decrease in PU.1 protein compared with control cells (Figure 4B).

To overexpress SATB1 we infected U937 cells with an IRESSATB1-GFP lentivirus, sorted $\mathrm{GFP}^{+}$cells, and assessed SATB1 as well as PU.1 mRNA levels by means of quantitative real-time RTPCR. While U937 cells infected with an empty control IRES-GFP lentivirus did not show a change of either SATB1 or PU.1 expression compared with uninfected controls, we found a 7.2-fold upregulation of SATB1 $(P<0.001)$ and a 3-fold upregulation of PU.1 $(P=0.01)$ in U937 cells treated with the SATB1 lentivirus (Figure 4C). Cells exposed to SATB1 lentivirus but GFP- (i.e., untransduced cells) did not show altered SATB1 or PU.1 expression.

We next asked whether this regulatory role for SATB1 is dependent on the URE. To address this question, we lentivirally overexpressed SATB1 in myeloid leukemic cells with a targeted disruption of the URE (URE-/-). In contrast to our observation in wild- type U937 cells (Figure 4C), we did not observe an upregulation of PU.1 expression levels in URE-/- cells (Figure 4D). Also, to address this issue in purified myeloid progenitor cells, we FACS sorted lineage marker-negative and kit-positive $\left(\mathrm{Lin}^{-} \mathrm{c}-\mathrm{kit}^{+}\right)$progenitors from the BM of $\mathrm{URE}^{-/-}$mice and wild-type littermates and infected them with SATB1-expressing lentivirus. While ectopic SATB1 expression led to a 2.2-fold $(P<0.05)$ increase of PU.1 expression in wild-type progenitors, we did not find this effect in URE-deficient progenitors (Figure 4, E and F). Next we stably transfected the SATB1-directed siRNA expression construct in URE-/- cells, which led to a 3-fold reduction of SATB1 protein levels, but we did not observe a reduction of RNA levels of PU.1 (Figure 4G). Taken together, these data demonstrate that SATB1 regulates the PU.1 gene and that this regulation is dependent on the URE.

The PU.1 regulatory function of SATB1 is development dependent. Next we asked whether the effect of SATB1 on PU.1 expression can also be seen in vivo using SATB1-null mice, which have been previously described (20). We harvested total wbc from fetal livers of 12-day-old embryos and measured PU.1 expression in wild-type and SATB $1^{-/-}$cells by quantitative RT-PCR. We could not detect a difference in PU.1 levels in total fetal liver wbc (Figure 5A). As unfractionated wbc represent a heterogeneous cellular mixture, we 

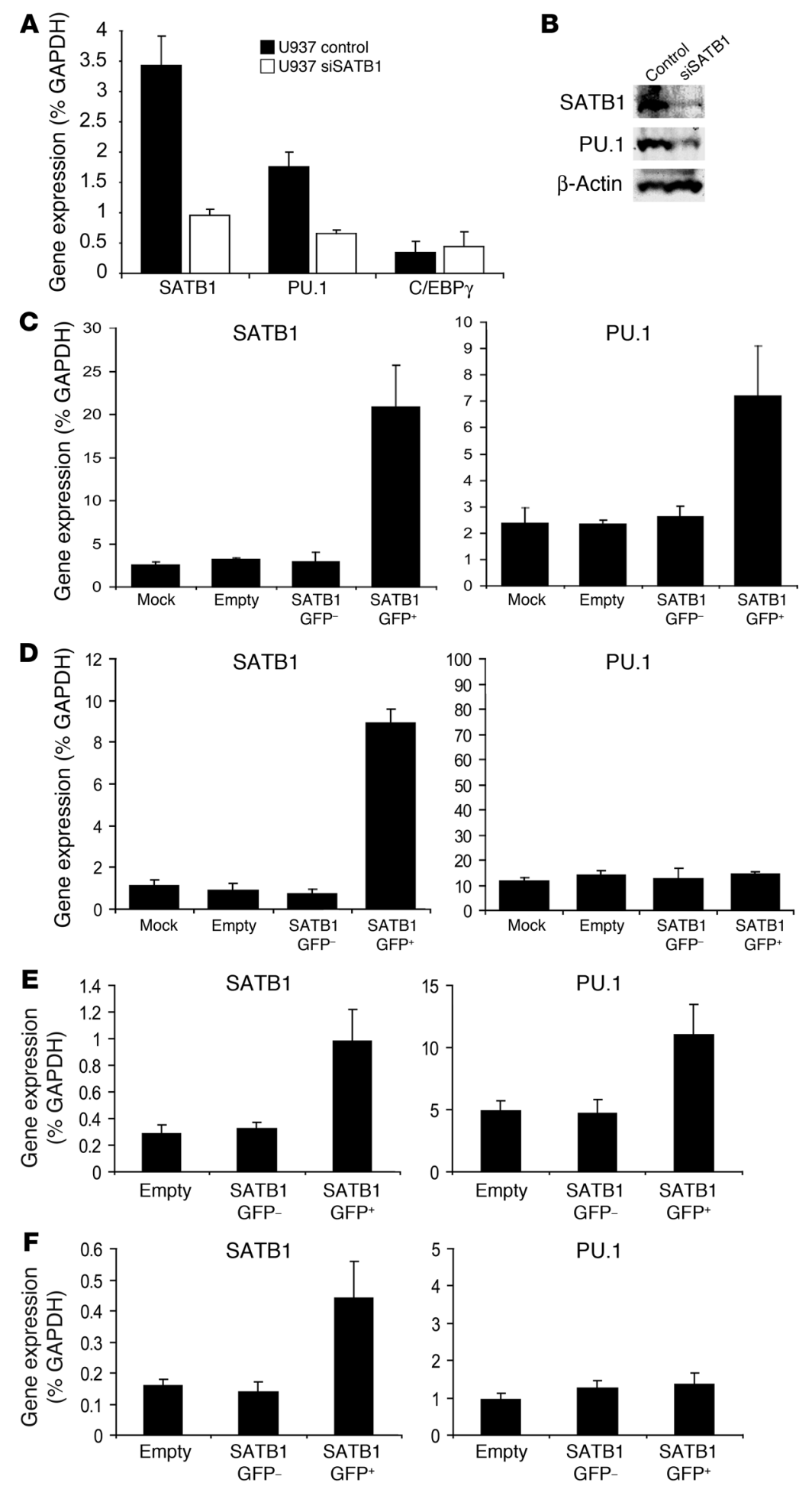

G

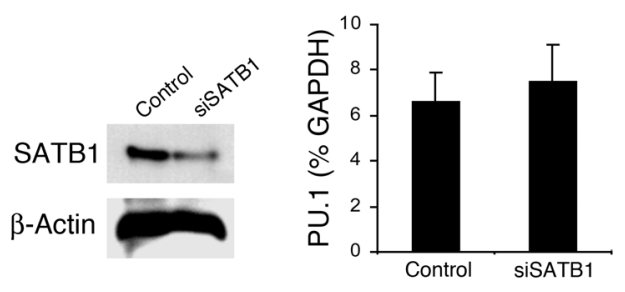

Figure 4

SATB1 is a URE-dependent positive regulator of PU.1 in myeloid cells. (A) SATB1-directed siRNAexpressing construct PU.1 mRNA was significantly reduced upon siRNA-mediated knockdown of SATB1 (U937 siSATB1). $n=3$. (B) Western blotting shows diminished PU.1 protein level after siRNA-mediated downregulation of SATB1 protein in stably transfected U937 cells. $\beta$-actin protein served as control. (C) Lentiviral overexpression of SATB1 in U937 cells leads to increased PU.1 expression. We utilized IRES-GFPSATB1 lentivirus to transduce U937 cells. GFP+ cells were FACS sorted and subjected to mRNA expression analysis. Sorted GFP- cells and cells infected with empty IRES-GFP lentivirus served as control. Gene expression data normalized to GAPDH. $n=3$. (D) SATB1 overexpression in the absence of URE does not lead to PU.1 upregulation. Cells derived from $\mathrm{URE}^{-/-}$mice were treated with SATB1-expressing lentivirus, GFP ${ }^{+}$and GFP- ${ }^{-}$cells FACS sorted, and SATB1 and PU.1 expression measured. While SATB1 expression was significantly increased in IRES-GFPSATB1-infected cells (SATB1 GFP+), there was no upregulation of PU. 1 expression in URE ${ }^{-/-}$cells. $n=3$. $(\mathbf{E}$ and $\mathbf{F})$ Lentiviral overexpression of SATB1 in sorted $\mathrm{Lin}^{-} \mathrm{Kit}^{+}$progenitors from wild-type littermates and URE-knockout mice. Lin-Kit ${ }^{+}$cells were FACS sorted and infected with empty control virus or IRES-GFPSATB1 virus. GFP+ cells were sorted and subjected to quantitative RT-PCR. (E) Sorted wild-type progenitors. $n=3$. (F) Sorted URE ${ }^{-/}$progenitors. $n=3$. (G) Neomycin resistance SATB1 siRNA expression construct was stably transfected into URE ${ }^{-/-}$cells and SATB1 and PU. 1 expression levels determined. An empty construct served as control. Mean \pm SD shown.

investigated phenotypically well defined stem and progenitor cell populations. Therefore we FACS sorted $\mathrm{Lin}^{-} \mathrm{c}-\mathrm{kit}{ }^{+} \mathrm{Sca} 1^{+} \mathrm{KLS}$ cells, $\mathrm{Lin}^{-} \mathrm{c}^{-} \mathrm{kit}^{+} \mathrm{Sca} 1^{-}$ CD34 $4^{\text {low }} \mathrm{F}_{c} \gamma \mathrm{II} / \mathrm{IIIR}{ }^{\text {low }}$ common myeloid progenitors (CMPs), Lin $^{-}$c-kit ${ }^{+}$Sca $1-\mathrm{CD} 34^{+} \mathrm{F}_{\mathrm{c}} \gamma \mathrm{II} / \mathrm{IIIR}^{+}$GMPs, and $\mathrm{Lin}^{-}{ }^{-}$- $\mathrm{kit}^{+} \mathrm{Sca} 1^{-} \mathrm{CD} 34^{-} \mathrm{F}_{\mathrm{c}} \gamma \mathrm{II} / \mathrm{IIIR}^{-}$megakaryocyte-erythrocyte progenitors (MEPs), representing developmentally earlier myeloid precursors and candidate leukemic stem cells in AML (reviewed in ref. 21). While we did not find a significant change of PU.1 expression in KLS cells or CMPs of SATB1-/ animals, PU. 1 expression was reduced by $88 \%$ in GMPs and by $80 \%$ in MEPs in comparison to wildtype littermates $(P<0.01)$ (Figure $5 \mathrm{~A})$. These data suggest that SATB1 functions as a positive regulator of PU.1 expression at specific points during myeloid development in vivo.

The homozygous SNP is associated with low PU.1 expression in progenitors of patients with AML. To address the question of whether the SNP affects PU.1 expression in human HSCs, we analyzed total BM specimens of patients with AML. While some patients carrying the homozygous SNP in the PU.1 enhancer had very low PU.1 levels, the average PU.1 expression was lower but not significantly different when compared with patients with 

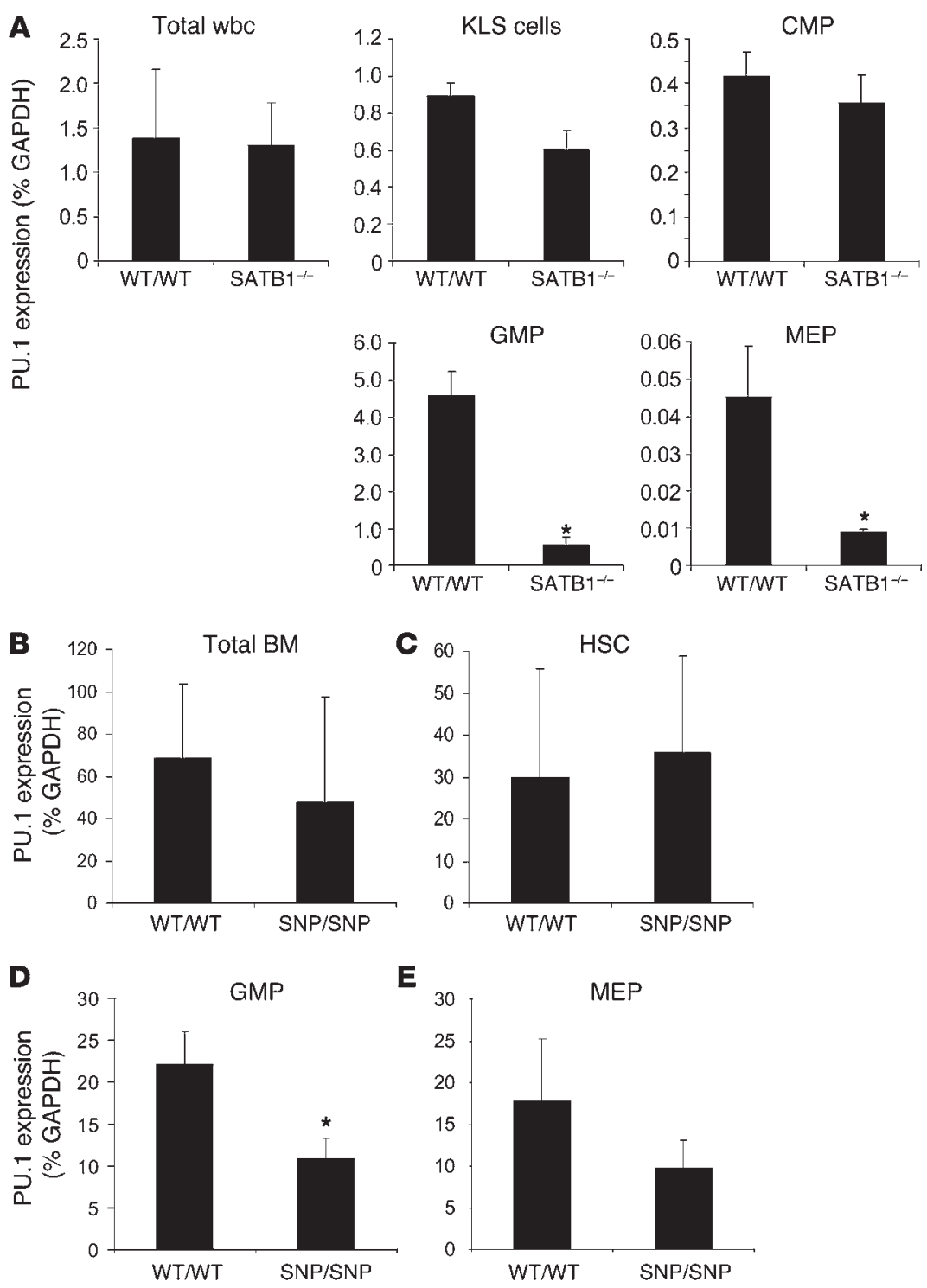

Figure 5

SATB1- and SNP-dependent downregulation of PU.1 in distinct myeloid progenitor subsets in vivo. (A) wbc from fetal livers of SATB1-knockout mice were harvested and KLS cells ( $\mathrm{Lin}^{-} \mathrm{c}-\mathrm{kit}^{+} \mathrm{Sca} 1^{+}$),

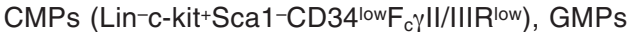
$\left(\mathrm{Lin}^{-} \mathrm{c}-\mathrm{kit}^{+} \mathrm{Sca} 1^{-\mathrm{CD} 34}{ }^{+} \mathrm{F}_{\mathrm{c}} \gamma \mathrm{ll} / \mathrm{IIIR} \mathrm{R}^{+}\right.$), and MEPs ( $\mathrm{Lin}^{-}$

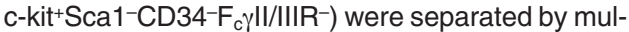
ticolor FACS sorting. PU.1 expression was determined by quantitative RT-PCR. GAPDH served as a control. SD is indicated by error bars $(n=3)$. While there are no significant changes in total wbc, KLS cells, or CMPs, PU.1 expression is markedly decreased in GMPs and MEPs of SATB1 $1^{-/}$mice in comparison with wild-type littermates. (B) Total BM of WT/WT $(n=16)$ and SNP/SNP $(n=5)$ patients was examined by quantitative RT-PCR. GAPDH expression served as a control. Averages and standard deviations (error bars) are shown. (C) Lin-CD34+CD38-Thy $1^{\text {low }}$ HSCs of WT/WT $(n=8)$ and SNP/SNP $(n=4)$ patients were separated by multicolor FACS and PU. 1 expression was determined by quantitative RT-PCR. (D) LinCD $34{ }^{+}$CD $38{ }^{+}$CD $123{ }^{+}$CD $45 R^{2}{ }^{+}$GMPs of WT/WT $(n=7)$ and SNP/SNP patients $(n=3)$ were FACS sorted and PU. 1 expression was measured by quantitative RT-PCR. Expression of GAPDH was used as a control. (E) Lin-CD34+CD38+CD123-CD45RAMEPs of WT/WT $(n=7)$ and SNP/SNP patients $(n=3)$ were FACS sorted and PU. 1 expression was measured by quantitative RT-PCR. Error bars represent standard deviation. Statistical significance is indicated by asterisks. $P<0.01$. the wild-type SATB1 site (Figure 5B). As unfractionated BM cells are a heterogeneous cellular population and we had already found in SATB1 ${ }^{-/}$mice that the SATB1-mediated regulation of PU.1 is restricted to certain cell types, we FACS sorted $\mathrm{Lin}^{-} \mathrm{CD} 34^{+} \mathrm{CD} 38^{-}$ Thy $1^{\text {low }}$ HSCs as well as $\mathrm{Lin}^{-} \mathrm{CD} 34^{+} \mathrm{CD} 38^{+} \mathrm{CD} 123^{+} \mathrm{CD} 45 \mathrm{RA}^{+}$ GMPs and $\mathrm{Lin}^{-} \mathrm{CD} 34^{+} \mathrm{CD} 38^{+} \mathrm{CD} 123^{-} \mathrm{CD} 45 \mathrm{RA}^{-}$MEPs of patients with AML. Enriched HSCs carrying the homozygous SNP did not have significantly different PU.1 expression than HSCs with the wild-type site (Figure 5C). Strikingly, GMPs of patients with the homozygous SNP showed 2-fold lower PU.1 levels in comparison with GMPs with the wild-type site in the PU.1 enhancer $(P<0.01)$ (Figure 5D). Also, MEPs with the homozygous SNP had 1.8-fold lower PU.1 expression $(P=0.15)$ than wild-type MEPs (Figure 5E). These findings show that the SNP leads to decreased PU.1 levels in distinct progenitor populations of patients with AML.

\section{Discussion}

The transcription factor PU.1 plays an important role in normal myeloid development. Recently it has been demonstrated that reduction of PU.1 levels by knockout of a highly conserved distal enhancer of PU.1 (URE) leads to critical transcriptional changes and ultimately to the development of AML in mice $(7,9)$. In humans mutations of the PU.1 gene are rare, but it has been shown that the fusion oncogenes PML-RARA and AML1-ETO inhibit PU.1 expression and protein function, respectively, in patients with AML (22-24). These findings suggest that reduced PU.1 contributes to the molecular pathogenesis of AML. However, the role of the URE of PU.1, the knockout of which induces AML in mice, has not been studied in human disease so far.

In this study we examined the highly conserved locus of the URE by FISH and direct sequencing in patients with AML. Using FISH, we did not find occult cytogenetic abnormalities, indicating that deletion or translocation of the URE are not frequent events in patients with AML. However, we found a SNP in the URE that, in its homozygous form, is more frequent in AML patients with complex karyotype. We cannot completely rule out very small deletions, as the resolution of FISH is limited. However, the fact that we did not find any samples with discrepancies between the SNP 
in hematopoietic and nonhematopoietic tissue speaks against the possibility that small deletions account for the detected homozygosity of the SNP. Since leukemic cells of URE-knockout mice show genetic instability and frequently carry cytogenetic abnormalities (7), we hypothesized that this SNP might play a role in the regulation of PU.1 and the pathogenesis of complex karyotypic AML in humans.

SATB1 is a chromatin-remodeling protein that was originally discovered in thymocytes and has been shown to regulate gene expression over long distances (17). We found that SATB1 binds to the URE of PU.1 in myeloid leukemic cells and that the SNP significantly diminishes binding of the SATB1 complex to the URE. We have shown previously that the PU.1 promoter alone is insufficient to drive PU.1 expression and that the distal URE in combination with the promoter is required $(15,16)$. Strikingly, the SNP in the URE led to a reduction of reporter gene expression, suggesting a role of SATB1 binding to the URE for the transcriptional activation of PU.1. Indeed, in overexpression and inhibition studies of SATB1 in myeloid leukemic cells, we found that inhibition of SATB1 led to a specific reduction of PU.1 RNA as well as protein. Conversely, overexpression of SATB1 caused an upregulation of PU.1 expression. These findings demonstrate that SATB1 is a positive regulator of PU.1. When we overexpressed or inhibited SATB1 in cells lacking the URE, PU.1 expression remained unchanged, indicating that SATB1 requires the URE to regulate PU.1 expression.

SATB1 can act as a distal regulator of gene expression in $\mathrm{T}$ cells by bringing multiple far distal target sequences together by tethering them to the cage-like SATB1 network, forming chromatin loop configuration, and recruiting chromatin-remodeling and transcription factors to the target genes $(17,18,20,25)$. The highly conserved PU.1-regulating URE is located $16 \mathrm{~kb}$ (human) and 14 $\mathrm{kb}$ (murine), upstream of the transcriptional start site of PU.1, suggesting that SATB1 has a similar long-range mechanism in myeloid cells. Several reports have shown a transcription-repressing function of SATB1 in T cells $(26,27)$. Recently, Wen et al. have shown that SATB1 positively regulates $\varepsilon$-globin gene expression in erythroid progenitor cells (28). Our data show that SATB1 can act as a transcriptional activator in myeloid cells. Apparently, SATB1 can be both a positive and negative transcriptional regulator depending on the cellular context and the target gene.

The finding that overexpression of SATB1 increases PU.1 and knockdown of SATB1 decreases PU.1 levels in U937 cells indicates that SATB1 regulates PU.1 in a dynamic range that is neither positively nor negatively saturated. This range suggests that modest changes in SATB1 function or binding in either direction might critically disturb the precisely regulated PU.1 expression levels in myeloid cells.

We could not detect a change in PU.1 levels in total wbc from SATB1 ${ }^{-/}$animals. However, we observed that PU.1 expression was reduced in SATB1 $1^{--}$GMPs and MEPs but not in KLS cells and CMPs. This finding indicates that the in vivo regulation of PU.1 by SATB1 is stage specific during myeloid differentiation. It also suggests that there is redundancy with regard to SATB1 function in certain cell types at certain stages of differentiation and that other factors might substitute for SATB1 in those cells.

Similarly, in total BM from patients with AML carrying the SNP, we found slightly but not significantly decreased PU.1 levels. However, when we FACS sorted and examined enriched HSCs, GMPs, and MEPs of patients with AML, we observed that GMPs carrying the SNP in the URE expressed lower PU.1 as compared with GMPs with the wild-type site. In contrast, we did not find significantly decreased PU.1 levels in stem cells. These data are analogous to our findings in SATB1/- mice and suggest that the PU.1-regulating effect of SATB1 is restricted to certain cell types, including GMPs and MEPs, in humans as well as mice. Interestingly, these data also support the necessity to analyze gene expression in AML at a cell type-specific level rather than investigating whole BM. The interpretation of gene expression data derived from unfractionated BM cells of patients with AML is difficult for several reasons. BM cells represent a heterogeneous cellular mixture, and thus the examination of genes whose expression changes during differentiation, such as developmental regulators like PU.1, is hampered due to great differences in maturation between different AML subtypes. Also, it is difficult to accurately measure levels of PU.1 and other regulators that are expressed at highest levels in mature cells because even a small number of granulocytes will confound the detection of decreased PU.1 in AML blast populations. We have recently demonstrated the advantage of comparing levels of PU.1 and its target genes, such as JunB, in FACS-sorted HSCs versus total BM (9).

Several groups have reported recently that experimental introduction of genetic modifications into different myeloid stem and progenitor subsets can lead to different functional effects including formation of leukemic stem cells, and thus, a specific cell type might need to be targeted by a certain oncogenic event in order to induce leukemia in vivo. In some experimental models of myeloid leukemia, this cell of origin appears to be the HSCs, while in others it is the GMP compartment (refs. 29, 30 and reviewed in ref. 21). However, the mechanisms of this stage specificity of oncogenic events are unclear. Our data point out the possibility that genetic factors such as the SNP in the enhancer of PU.1 can be present but functionally silent at the stem cell level and become phenotypically relevant at later stages during myeloid development, including GMPs, where lack of SATB1-mediated PU.1 regulation may cause a block of myeloid differentiation.

Of note, unlike $\mathrm{URE}^{-/-}$mice, SATB1/-- mice do not develop leukemia. SATB1 $1^{-/}$animals have a block in T cell development and normally die a few weeks after birth, which might be too early for myeloid leukemia to develop. It could also be that reduced PU.1 at the GMP and MEP stages is not sufficient as a sole cause for leukemia development and that effects in the KLS cell compartment are critical for PU.1 knockdown-induced leukemia. Interestingly, in humans the SNP in the URE does also not seem to act as a leukemia-initiating factor but rather as a modifier. This might again be due to the fact the SNP affects PU.1 levels in progenitors but not in HSCs. This might be sufficient to contribute to the development of genomic instability but not for formation of overt leukemia, in which decreased levels of PU.1 in earlier developmental stages might be required.

AML with complex karyotypes display an adverse prognosis with lower primary response and higher relapse rates compared with other cytogenetic risk groups, translating into poor overall survival despite the use of different treatment options including intensive therapy regimens. Even upon allogeneic HSC transplantation, the most aggressive antileukemic treatment, long-term survival is rare due to relapse and treatment-related complications (31-34). The biology of AML with complex karyotypes is still very poorly understood, but it has recently been suggested that altered DNA repair may play an important role in the generation of complex genetic aberrations (35). Our data suggest that the SNP in 
the PU.1 URE and consequential PU.1 downregulation may facilitate generation of complex-aberrant GMP cell clones and progression to AML with complex karyotype. Importantly, in our patient cohort the homozygous SNP was more frequent in patients with complex karyotype in comparison with normal karyotype, but not in all AML patients when compared with normal control subjects. This finding suggests a role in leukemia progression, in that the SNP acts as a modifier and favors a specific AML subtype, complex karyotypic AML, rather than a leukemia-initiating effect per se. It is possible that the observed association is even stronger in a more rigorously defined subgroup of AML patients. This has to be investigated in larger clinical cohorts. Also, the potential clinical importance of this association, especially the impact on survival, needs to be tested in large homogeneously treated clinical cohorts, allowing for multivariate analyses.

An increasing number of SNPs in proximal promoters and introns have recently been linked with diseases. For instance, SNPs in the promoters of the TBX21 and eotaxin 1 genes and SNPs in the promoter and introns of the STAT4 gene have been shown to be associated with asthma (36-38). A SNP in the proximal promoter of the TCOF1 gene was found to be associated with and functionally relevant in Treacher Collins Syndrome, an autosomal-dominant craniofacial malformation (39). A SNP in intron 4 of the ZDHHC8 gene showed a strong association with susceptibility to schizophrenia (40). Also, intronic SNPs in the PDCD1 and SLC224A genes have been found to alter binding of RUNX1 and are associated with rheumatoid arthritis and systemic lupus erythematodes, respectively $(41,42)$. In a recent study of $\alpha$-thalassemia, a SNP was identified that creates a new transcriptional promoter-like element that activates expression of aberrant transcripts and disrupts physiologic $\alpha$-globin expression (43). The results of these and other studies provide increasing evidence for the high functional significance that solitary SNPs may have.

Here we show for the first time that a SNP in a distal enhancer many kilobases upstream of the coding sequence is associated with a subtype of leukemia in humans. Moreover, our study provides novel mechanistic insights in that the SNP disrupts physiologic regulation of tumor suppressor gene expression at distinct stages during myeloid development, an effect that is mediated by reduced binding of a chromatin-remodeling protein that can act over long distances. Our findings demonstrate that not only may SNPs within coding sequences and proximal promoter regions of genes be functionally important, but SNPs in far distal regulatory elements might also be critical for transcriptional regulation of tumor suppressors and thus development of cancer.

\section{Methods}

Human samples. After receiving written informed consent, cells were derived from patients with AML in the context of routine diagnostic BM punctures and from healthy volunteers. The male/female ratio of the AML patients was 0.81:1. The subclassification of AMLs according to FrenchAmerican-British group criteria was as follows: M0 11x, M1 23x, M2 39x, M3 6x, M4 43x, M5 9x, M6 8x, and M7 2x. Thirty-eight patients with AML with a previous history of myelodysplastic syndrome and 20 therapy-associated AMLs have been included. All patients and controls were Caucasian. In all patients classical cytogenetics was performed, comprising 101 patients with normal karyotypes, 77 with complex karyotypes (with 3 or more abnormalities), 4 patients with sole monosomy 7, 9 patients with trisomy 8,10 patients with translocation $\mathrm{t}(8 ; 21), 9$ patients with inversion inv(16), 3 patients with translocation $\mathrm{t}(15 ; 17)$, and 23 patients with other miscellaneous cytogenetic abnormalities. The study was approved by the Institutional Review Board of Beth Israel Deaconess Medical Center.

Mice. SATB1-knockout mice as well as PU.1-knockdown mice with targeted disruption of the URE of the PU.1 gene have been previously described $(7,20)$. Mouse experiments were approved by the Beth Israel Deaconess Medical Center Institutional Animal Care and Use Committee.

FISH. Locus-specific interphase and metaphase fluorescence in situ hybridization was performed on BM cells following short-term culture according to standard protocols for classic cytogenetics. Bacterial artificial chromosome clones RP11-379M04 and RP11-17G12 (Children's Hospital Oakland Research Institute, Oakland, California, USA) were selected to cover the first and second homology region (RP11-379M04) and the homology regions plus the PU.1 locus (RP11-17G12) (Figure 1A). Bacterial cultivation and bacterial artificial chromosome DNA isolation, labeling, probe preparation, and hybridization of the slides were performed as previously described (44).

Sequencing. DNA was isolated from BM samples by Qiagen extraction kit (Qiagen). We used the following 2 primer pairs (URE-1 and URE-2) to amplify both homology regions of the URE: URE-1 (forward), GCTGTTGGGTGTCCAGGG; URE-1 (reverse), CACCTTGCCTTGGGGAGG; URE-2 (forward), AGAAGAAGGCTGAGGCCTGAGGCC; URE-2 (reverse), AACTCGGGCCACCACTGCTTGG. PCR was carried out in a final volume of $50 \mu \mathrm{L}$ containing genomic DNA (100 ng). Sequencing was performed in both directions. Subsequent gene scanning and sequence analysis was performed using an ABI 3130 Genetic Analyzer and Sequencing Analyzing software version 5.2 (Applied Biosystems) and using manual assessment of sequencing traces. Sequences with an abnormal result or the detected SNP were controlled by an independent second sequencing.

Statistics. Results of the sequence analyses were tested for statistical significance by standard $\chi^{2}$ test and Fisher's exact test utilizing the Statistica 6.1 software (StatSoft Inc.). Statistical significance of overexpression and inhibition studies was checked by 2 -tailed Student's $t$ test. $P$ values smaller than 0.05 were considered statistically significant.

Chromatin immunoprecipitation assay for in vivo DNA binding. Chromatin immunoprecipitation experiments were performed as previously described (16). Chromatin was isolated from myeloid U937 cells and sonicated 3 times for 10 seconds with a $90 \%$ duty cycle and output setting 4 on a Branson Sonifier 450 apparatus. Immunoprecipitation was performed with $10 \mu \mathrm{g}$ of SATB1 antibody (Santa Cruz Biotechnology Inc.) or $10 \mu \mathrm{g}$ of normal rabbit IgG (Santa Cruz Biotechnology Inc.). For PCR of the first homology region of the URE, the following oligonucleotides were used: forward, $5^{\prime}$-CCCAGGCAAGGGAAGTTTGT-3' and reverse, 5'-CCTCTTGCTTCTGGTCCCC-3'. Primers for the known SATB1 binding sites SBS336 and SBS700 served as positive controls and have been described previously (17).

EMSA for in vitro DNA binding. EMSA was performed as previously described (16). The following oligonucleotides were annealed and used as probes: wild-type probe, $5^{\prime}$-CTTTGATTTATTATAGCCATGAAAT-3' and 5'-ATTTCATGGCTATAATAAATCAAAG-3'; SNP probe, 5'-CTTTGATTTATTAGAGCCATGAAAT-3' and $5^{\prime}$-ATTTCATGGCTCTAAATCAAAG-3'; SATB1 IgH site, 5' -TCTTTAATTTCTAATATATTTAGAA-3' and 5'-TTCTAAATATATTAGAAATTAAAGA-3'; Sp1 probe, 5'-AAACGGCTGGGGGCGGTGATGTCAC-3' and 5'-GTGACATCACCGCCCCAGCCCGTTT-3'. Annealed oligonucleotides were labeled with $\left[\gamma^{32} \mathrm{P}\right]$ ATP using T4 polynucleotide kinase. They were then gel purified utilizing $10 \%$ polyacrylamide gel. Probes were incubated with nuclear extracts of myeloid U937 cells in $10 \mathrm{mM}$ HEPES (pH 7.8), $50 \mathrm{mM} \mathrm{KCl}, 1 \mathrm{mM}$ dithiothreitol, $1 \mathrm{mM}$ EDTA, and $5 \%$ glycerol for 30 minutes. Reaction mixtures were separated with $6 \%$ polyacrylamide gels in $0.5 \times \mathrm{TBE}$ buffer at $4^{\circ} \mathrm{C}$. PU. 1 antibody (Santa Cruz Biotechnology Inc.) and C/EBP $\alpha$ antibody (used as a control; Santa Cruz Biotechnology Inc.) were used for supershift assays. 
Enhancer activity assays. Constructs including the $0.5-\mathrm{kb}$ PU.1 promoter and the PU.1 URE cloned into the PXP2 luciferase vector have been described elsewhere $(15,16)$. To introduce the point mutation representing the SNP of the first homology region into the URE, PCR mutagenesis was performed as previously described (45). The mutated URE was verified by sequencing. For isolation of stable transformants and luciferase assays, U937 cells were cultured with RPMI, 10\% FCS, and transfected by Lipofectamine with $2 \mu \mathrm{g}$ of linearized reporter construct and $0.1 \mu \mathrm{g}$ pGKneo per $25-\mathrm{cm}^{2}$ flask. Stable transformants were selected by addition of $1 \mathrm{mg} / \mathrm{ml}$ of G418 for 2 weeks beginning 48 hours after transfection. Independent clones of each construct were obtained by limiting dilution in 96-well plates. Luciferase assays were performed utilizing the BrightGlo Luciferase Assay System (Promega) according to the manufacturer's instructions. Luciferase activity was standardized by the construct copy number of each clone as determined by Southern blotting using the 0.5 -kb PU.1 promoter fragment as a probe.

siRNA-mediated knockdown of SATB1. For stable inhibition of SATB1, we utilized a pSUPER vector (OligoEngine Inc.) encoding a SATB1-directed siRNA and including a neomycin resistance cassette. This construct was transfected into U937 and $\mathrm{URE}^{-/-}$cells, and stable transformants were obtained by selection with $2 \mathrm{mg} / \mathrm{ml} \mathrm{G} 418$. An empty pGKneo construct served as a control.

Lentiviral SATB1 expression experiments. We created a SATB1-expressing lentivirus by introducing the SATB1 coding sequence into the EcoRI site of a pCAD-IRES-GFP lentiviral construct. We treated U937 cells, URE-/leukemic cells, and FACS-sorted $\mathrm{Lin}^{-} \mathrm{Kit}^{+} \mathrm{BM}$ cells from $\mathrm{URE}^{-/-}$mice with the empty virus (IRES-GFP) and the IRES-GFP-SATB1 lentivirus as previously described (9). In brief, we cultured U937 cells in RPMI, 10\% FCS, and infected them by adding concentrated cell-free lentiviral supernatants at an MOI of 10 for 48 hours in the presence of $8 \mu \mathrm{g} / \mathrm{ml}^{-1}$ polybrene. URE-/leukemic cells were grown in Myelocult M5300 (Stem Cell Technologies) containing 10\% FCS and 5\% WEHI supernatant before and during treatment with the lentivirus.

FACS-sorted Lin-Kit ${ }^{+}$cells were cultured in CellGenix SCGM media supplemented with SCF (100 ng/ml), Flt3-L (100 ng/ml), Tpo (50 ng/ml), IL-3 $(20 \mathrm{ng} / \mathrm{ml})$, and IL- $6(20 \mathrm{ng} / \mathrm{ml})$ and double transfected (after 6 hours and 30 hours) with lentivirus. After 3 washing steps in complete medium, and 72 hours after transduction, we sorted $\mathrm{GFP}^{+}$and GFP- cells utilizing a high-speed cell sorter (MoFlo-MLS; Cytomation).

Quantitative real-time RT-PCR. We extracted total RNA from stable cell lines (siRNA inhibition experiments) or FACS-sorted GFP' and GFP- cells (overexpression experiments) using RLT buffer and $20 \mathrm{ng}$ bacterial carrier RNA (Roche Diagnostics) per sample according to the RNeasy micro protocol (Qiagen) optimized for small amounts of RNA. RNA was treated with DNAse I according to the manufacturer's instructions. We amplified the resultant RNA utilizing the TaqMan One-Step RT-PCR Master Mix (Applied Biosystems) and an ABIPrism 7700 Sequence Detector (Applied Biosystems) with 1 cycle each of $48^{\circ} \mathrm{C}(30$ minutes $)$ and $95^{\circ} \mathrm{C}(10 \mathrm{~min}$ utes) followed by 40 cycles of $95^{\circ} \mathrm{C}\left(15\right.$ seconds), $60^{\circ} \mathrm{C}$ ( 1 minute), and $72^{\circ} \mathrm{C}$ (1 minute). The gene expression assays for SATB1, PU.1, C/EBP $\gamma$, and GAPDH, each consisting of a validated pre-made primer/probe set (Applied Biosystems), were used for detection and quantification of SATB1, PU.1, $\mathrm{C} / \mathrm{EBP} \gamma$, and GAPDH, respectively, as controls.

Western blot assays. We extracted total cell lysates as previously described (46). Proteins were resolved by SDS-PAGE and electrotransferred to a nitrocellulose membrane (Bio-Rad). We used polyclonal rabbit antibody to SATB1 (Santa Cruz Biotechnology Inc.), monoclonal goat antibody to PU.1 (Santa Cruz Biotechnology Inc.), and monoclonal mouse antibody to $\beta$-tubulin (Sigma-Aldrich). We detected immunoreactive proteins using HPRT-conjugated antibodies to mouse, rabbit, or goat (Santa Cruz Biotechnology Inc.) and the ECL system (Amersham Biosciences). Bands were quantified using ImageQuant densitometry software (Amersham Biosciences).

Flow cytometry and sorting of HSCs and progenitor cells. Murine fetal liver cells of SATB1 1 - embryos (day 12) were analyzed on a FACScan cytometer (BD) by gating on viable cells by exclusion of propidium iodide staining. After lysis of erythrocytes, lineage depletion of BM cells was accomplished using rat anti-mouse antibodies directed against CD3, CD4, CD8a, CD19, Ly-6G, Ter119, and CD45R antigens. Flow cytometric sorting of Lin $^{-}{ }^{-}-k_{i t}{ }^{+} \mathrm{Sca}-1^{+}$ KLS cells using a double laser $\left(488 \mathrm{~nm} / 350 \mathrm{~nm}\right.$ Enterprise II ${ }^{+} 647 \mathrm{Spec}-$ trum) high-speed cell sorter (MoFlo-MLS; Cytomation) has been described previously (47). Lin $^{-}$c-kit ${ }^{+}$Sca1 ${ }^{-} \mathrm{CD} 34^{\text {low }} \mathrm{F}_{\mathrm{c}} \gamma \mathrm{II} / \mathrm{IIIR}^{\text {low }} \mathrm{CMPs}$, Lin ${ }^{-} \mathrm{c}-\mathrm{kit}^{+} \mathrm{Sca}{ }^{-}$ $\mathrm{CD}^{3} 4^{+} \mathrm{F}_{c} \gamma \mathrm{II} / \mathrm{IIIR}^{+} \mathrm{GMPs}$, and $\mathrm{Lin}^{-} \mathrm{c}-\mathrm{kit}^{+} \mathrm{Sca} 1^{-} \mathrm{CD} 344^{-} \mathrm{F}_{c} \gamma \mathrm{II} / \mathrm{IIIR}^{-}$MEPs were also separated by multicolor FACS sorting as described (47).

Human HSCs and myeloid progenitors were isolated from BM of patients with AML as reported previously $(48,49)$. In brief, CD34+ cells from $\mathrm{BM}$ mononuclear cells were enriched utilizing immunomagnetic beads as previously described $(50,51)$. CD34 $4^{+}$cells were then stained with phycoerythrin-Cy5-conjugated antibodies directed against lineage antigens as well as CD34-APC, Thy1-FITC, and CD38-APC-Cy7 antibodies. Viable Lin ${ }^{-} \mathrm{CD} 34^{+} \mathrm{CD} 38^{-}$-Thy $1^{\text {low }}$ cells (HSCs) were sorted by a MoFlo-MLS cell sorter (Cytomation). For separation of GMPs and MEPs, cells were stained with lineage antibodies CD34-APC, CD38-APC-Cy7, CD45RA-FITC, and CD123-PE and then high-speed sorted (GMPs: Lin ${ }^{-}$ CD $34^{+} \mathrm{CD} 38^{+} \mathrm{CD} 123^{+} \mathrm{CD} 45 \mathrm{RA}^{+}$; MEPs: Lin $\left.{ }^{-} \mathrm{CD} 34^{+} \mathrm{CD} 38^{+} \mathrm{CD} 123^{-} \mathrm{CD} 45 \mathrm{RA}\right)$ as previously described (48). Purity of the sorted cell populations ranged between $97 \%$ and $99.4 \%$.

\section{Acknowledgments}

We thank Chris Hetherington for quantitative real-time RT-PCR analysis and John Tigges and Vasilis Toxavidis for expert assistance with multicolor flow cytometry and high-speed cell sorting. We thank Bruce Torbett for providing us with the pCAD-IRES-GFP lentivirus. We thank Steffen Klippel, Boris Bartholdy, Constantine Mitsiades, Stefan Fröhling, and Claudia Scholl for helpful discussion. U. Steidl thanks Sandra Steidl for invaluable support and advice. This work was supported by NIH grant CA41456 to D.G. Tenen and by fellowships from the Dr. Mildred Scheel Foundation for Cancer Research (to U. Steidl; D/03/41221), the German Research Foundation (to C. Steidl; STE1706/1-1), the Lymphoma Research Foundation (to F. Rosenbauer), and the German Research Foundation (to S. Koschmieder; KO2155/1-1), and by a scholarship from the German José Carreras Leukemia Foundation to B. Will.

Received for publication October 2, 2006, and accepted in revised form May 14, 2007.

Address correspondence to: Daniel G. Tenen, Harvard Institutes of Medicine, HIM Building, Room 954, 77 Avenue Louis Pasteur, Boston, Massachusetts 02115, USA. Phone: (617) 667-5561; Fax: (617) 667-3299; E-mail: dtenen@bidmc.harvard.edu.

Ulrich Steidl and Christian Steidl contributed equally to this work.
1. Scott, E.W., Simon, M.C., Anastasi, J., and Singh, H. 1994. Requirement of transcription factor PU.1 in the development of multiple hematopoietic lineages. Science. 265:1573-1577.
2. McKercher, S.R., et al. 1996. Targeted disruption of the PU.1 gene results in multiple hematopoietic abnormalities. EMBO J. 15:5647-5658.

3. Iwasaki, H., et al. 2005. Distinctive and indispens- able roles of PU. 1 in maintenance of hematopoietic stem cells and their differentiation. Blood. 106:1590-1600.

4. Nutt, S.L., Metcalf, D., D’Amico, A., Polli, M., and 
Wu, L. 2005. Dynamic regulation of PU.1 expression in multipotent hematopoietic progenitors. J. Exp. Med. 201:221-231.

5. Tenen, D.G., Hromas, R., Licht, J.D., and Zhang, D.E. 1997. Transcription factors, normal myeloid development, and leukemia. Blood. 90:489-519.

6. Tenen, D.G. 2003. Disruption of differentiation in human cancer: AML shows the way. Nat. Rev. Cancer. 3:89-101.

7. Rosenbauer, F., et al. 2004. Acute myeloid leukemia induced by graded reduction of a lineage-specific transcription factor, PU.1. Nat. Genet. 36:624-630.

8. Rosenbauer, F., et al. 2006. Lymphoid cell growth and transformation are suppressed by a key regulatory element of the gene encoding PU.1. Nat. Genet. 38:27-37.

9. Steidl, U., et al. 2006. Essential role of Jun family transcription factors in PU.1-induced leukemic stem cells. Nat. Genet. 38:1269-1277.

10. Rosenbauer, F., Koschmieder, S., Steidl, U., and Tenen, D.G. 2005. Effect of transcription-factor concentrations on leukemic stem cells. Blood. 106:1519-1524.

11. Cook, W.D., et al. 2004. PU.1 is a suppressor of myeloid leukemia, inactivated in mice by gene deletion and mutation of its DNA binding domain. Blood. 104:3437-3444.

12. Walter, M.J., et al. 2005. Reduced PU.1 expression causes myeloid progenitor expansion and increased leukemia penetrance in mice expressing PML-RARalpha. Proc. Natl. Acad. Sci. U. S. A. 102:12513-12518.

13. Dakic, A., et al. 2005. PU.1 regulates the commitment of adult hematopoietic progenitors and restricts granulopoiesis. J. Exp. Med. 201:1487-1502.

14. Suraweera, N., et al. 2005. Mutations of the PU.1 Ets domain are specifically associated with murine radiation-induced, but not human therapy-related, acute myeloid leukaemia. Oncogene. 24:3678-3683.

15. Li, Y., et al. 2001. Regulation of the PU.1 gene by distal elements. Blood. 98:2958-2965.

16. Okuno, Y., et al. 2005. Potential autoregulation of transcription factor PU.1 by an upstream regulatory element. Mol.Cell. Biol. 25:2832-2845.

17. Yasui, D., Miyano, M., Cai, S., Varga-Weisz, P., and Kohwi-Shigematsu, T. 2002. SATB1 targets chromatin remodelling to regulate genes over long distances. Nature. 419:641-645.

18. Cai, S., Han, H.J., and Kohwi-Shigematsu, T. 2003. Tissue-specific nuclear architecture and gene expression regulated by SATB1. Nat. Genet. 34:42-51.

19. Dickinson, L.A., Joh, T., Kohwi, Y., and KohwiShigematsu, T. 1992. A tissue-specific MAR/SAR DNA-binding protein with unusual binding site recognition. Cell. 70:631-645.

20. Alvarez, J.D., et al. 2000. The MAR-binding protein SATB1 orchestrates temporal and spatial expression of multiple genes during T-cell development. Genes Dev. 14:521-535.

21. Wang, J.C., and Dick, J.E. 2005. Cancer stem cells: lessons from leukemia. Trends Cell Biol. 15:494-501. 22. Mueller, B.U., et al. 2002. Heterozygous PU.1 mutations are associated with acute myeloid leukemia. Blood. 100:998-1007.

23. Vangala, R.K., et al. 2003. The myeloid master regulator transcription factor PU.1 is inactivated by AML1-ETO in t $(8 ; 21)$ myeloid leukemia. Blood. 101:270-277.

24. Mueller, B.U., et al. 2006. ATRA resolves the differentiation block in $\mathrm{t}(15 ; 17)$ acute myeloid leukemia by restoring PU.1 expression. Blood. 107:3330-3338.

25. Cai, S., Lee, C.C., and Kohwi-Shigematsu, T. 2006. SATB1 packages densely-looped, transcriptionally-active chromatin for coordinated expression of cytokine genes. Nat. Genet. 38:1278-1288.

26. Kohwi-Shigematsu, T., Maass, K., and Bode, J. 1997. A thymocyte factor SATB1 suppresses transcription of stably integrated matrix-attachment region-linked reporter genes. Biochemistry. 36:12005-12010.

27. Liu, J., et al. 1997. The matrix attachment regionbinding protein SATB1 participates in negative regulation of tissue-specific gene expression. $\mathrm{Mol}$. Cell. Biol. 17:5275-5287.

28. Wen, J., et al. 2005. SATB1 family protein expressed during early erythroid differentiation modifies globin gene expression. Blood. 105:3330-3339.

29. Huntly, B.J., et al. 2004. MOZ-TIF2, but not BCR$\mathrm{ABL}$, confers properties of leukemic stem cells to committed murine hematopoietic progenitors. Cancer Cell. 6:587-596.

30. Passegue, E., Wagner, E.F., and Weissman, I.L. 2004. JunB deficiency leads to a myeloproliferative disorder arising from hematopoietic stem cells. Cell. 119:431-443

31. Byrd, J.C., et al. 2002. Pretreatment cytogenetic abnormalities are predictive of induction success, cumulative incidence of relapse, and overall survival in adult patients with de novo acute myeloid leukemia: results from Cancer and Leukemia Group B (CALGB 8461). Blood. 100:4325-4336.

32. Schoch, C., et al. 2001. Patients with de novo acute myeloid leukaemia and complex karyotype aberrations show a poor prognosis despite intensive treatment: a study of 90 patients. Br. J. Haematol. 112:118-126.

33. Slovak, M.L., et al. 2000. Karyotypic analysis predicts outcome of preremission and postremission therapy in adult acute myeloid leukemia: a Southwest Oncology Group/Eastern Cooperative Oncology Group study. Blood. 96:4075-4083.

34. Grimwade, D., et al. 1998. The importance of diagnostic cytogenetics on outcome in AML: analysis of 1,612 patients entered into the MRC AML 10 trial. The Medical Research Council Adult and Children's Leukaemia Working Parties. Blood. 92:2322-2333.

35. Schoch, C., et al. 2005. Acute myeloid leukemia with a complex aberrant karyotype is a distinct biological entity characterized by genomic imbalances and a specific gene expression profile. Genes
Chromosomes Cancer. 43:227-238.

36. Akahoshi, M., et al. 2005. Functional promoter polymorphism in the TBX21 gene associated with aspirin-induced asthma. Hum. Genet. 117:16-26.

37. Chang, H.S., et al. 2005. A single nucleotide polymorphism on the promoter of eotaxin 1 associates with its mRNA expression and asthma phenotypes. J. Immunol. 174:1525-1531.

38. Park, B.L., et al. 2005. Association analysis of signal transducer and activator of transcription 4 (STAT4) polymorphisms with asthma. J. Hum. Genet. 50:133-138.

39. Masotti, C., et al. 2005. A functional SNP in the promoter region of TCOF1 is associated with reduced gene expression and YY1 DNA-protein interaction. Gene. 359:44-52.

40. Mukai, J., et al. 2004. Evidence that the gene encoding ZDHHC8 contributes to the risk of schizophrenia. Nat. Genet. 36:725-731.

41. Tokuhiro, S., et al. 2003. An intronic SNP in a RUNX1 binding site of SLC22A4, encoding an organic cation transporter, is associated with rheumatoid arthritis. Nat. Genet. 35:341-348.

42. Prokunina, L., et al. 2002. A regulatory polymorphism in PDCD1 is associated with susceptibility to systemic lupus erythematosus in humans. Nat. Genet. 32:666-669.

43. De Gobbi, M., et al. 2006. A regulatory SNP causes a human genetic disease by creating a new transcriptional promoter. Science. 312:1215-1217.

44. Steidl, C., et al. 2002. Patterns of chromosoma aberrations in urinary bladder tumours and adjacent urothelium. J. Pathol. 198:115-120.

45. Kobayashi, S., et al. 2005. EGFR mutation and resistance of non-small-cell lung cancer to gefitinib. N. Engl. J. Med. 352:786-792.

46. Kobayashi, S., et al. 2002. Calpain-mediated X-linked inhibitor of apoptosis degradation in neutrophil apoptosis and its impairment in chronic neutrophilic leukemia. J. Biol. Chem. 277:33968-33977.

47. Akashi, K., Traver, D., Miyamoto, T., and Weissman, I.L. 2000. A clonogenic common myeloid progenitor that gives rise to all myeloid lineages. Nature. 404:193-197.

48. Manz, M.G., Miyamoto, T., Akashi, K., and Weissman, I.L. 2002. Prospective isolation of human clonogenic common myeloid progenitors. Proc. Natl. Acad. Sci. U. S. A. 99:11872-11877.

49. Jamieson, C.H., et al. 2004. Granulocyte-macrophage progenitors as candidate leukemic stem cells in blast-crisis CML. N. Engl. J. Med. 351:657-667.

50. Steidl, U., et al. 2002. Gene expression profiling identifies significant differences between the molecular phenotypes of bone marrow-derived and circulating human CD34+ hematopoietic stem cells. Blood. 99:2037-2044.

51. Steidl, U., et al. 2004. Primary human CD34+ hematopoietic stem and progenitor cells express functionally active receptors of neuromediators. Blood. 104:81-88. 